\title{
Sialic acid receptor detection in the human respiratory tract: evidence for widespread distribution of potential binding sites for human and avian influenza viruses

\author{
John M Nicholls*1, Anthony J Bourne ${ }^{2}$, Honglin Chen ${ }^{3}$, Yi Guan ${ }^{3}$ and JS \\ Malik Peiris ${ }^{3}$
}

Address: 1Pathology Department, The University of Hong Kong, Pok Fu Lam, Hong Kong, Hong Kong SAR, 2Pathology Department, Adelaide Women and Children's Hospital, Adelaide, South Australia 5006, Australia and ${ }^{3}$ Microbiology Department, The University of Hong Kong, Pok Fu Lam, Hong Kong, Hong Kong SAR

Email: John M Nicholls* - nicholls@pathology.hku.hk; Anthony J Bourne - tbourne@internode.on.net; Honglin Chen - hlchen@hkucc.hku.hk; Yi Guan - yguan@hkucc.hku.hk; JS Malik Peiris - malik@hkucc.hku.hk

* Corresponding author

Published: 25 October 2007

Respiratory Research 2007, 8:73 doi:10.1 186/1465-992I-8-73
Received: 24 April 2007

Accepted: 25 October 2007

This article is available from: http://respiratory-research.com/content/8/I/73

(c) 2007 Nicholls et al; licensee BioMed Central Ltd.

This is an Open Access article distributed under the terms of the Creative Commons Attribution License (http://creativecommons.org/licenses/by/2.0), which permits unrestricted use, distribution, and reproduction in any medium, provided the original work is properly cited.

\begin{abstract}
Background: Influenza virus binds to cell receptors via sialic acid (SA) linked glycoproteins. They recognize SA on host cells through their haemagglutinins $(H)$. The distribution of SA on cell surfaces is one determinant of host tropism and understanding its expression on human cells and tissues is important for understanding influenza pathogenesis. The objective of this study therefore was to optimize the detection of $\alpha 2,3$-linked and $\alpha 2,6$-linked SA by lectin histochemistry by investigating the binding of Sambucus nigra agglutinin (SNA) for SA $22,6 \mathrm{Gal}$ and Maackia amurensis agglutinin (MAA) for $\mathrm{SA} \alpha 2,3 \mathrm{Gal}$ in the respiratory tract of normal adults and children.

Methods: We used fluorescent and biotinylated SNA and MAA from different suppliers on archived and prospectively collected biopsy and autopsy specimens from the nasopharynx, trachea, bronchus and lungs of fetuses, infants and adults. We compared different methods of unmasking for tissue sections to determine if these would affect lectin binding. Using serial sections we then compared the lectin binding of MAA from different suppliers.

Results: We found that unmasking using microwave treatment in citrate buffer produced increased lectin binding to the ciliated and glandular epithelium of the respiratory tract. In addition we found that there were differences in tissue distribution of the $\alpha 2,3$ linked SA when 2 different isoforms of MAA (MAAI and MAA2) lectin were used. MAAI had widespread binding throughout the upper and lower respiratory tract and showed more binding to the respiratory epithelium of children than in adults. By comparison, MAA2 binding was mainly restricted to the alveolar epithelial cells of the lung with weak binding to goblet cells. SNA binding was detected in bronchial and alveolar epithelial cells and binding of this lectin was stronger to the paediatric epithelium compared to adult epithelium. Furthermore, the MAA lectins from 2 suppliers (Roche and EY Labs) tended to only bind in a pattern similar to MAAI (Vector Labs) and produced a different binding pattern to MAA2 from Vector Labs.

Conclusion: The lectin binding pattern of MAA may vary depending on the supplier and the different isoforms of MAA show a different tissue distribution in the respiratory tract. This finding is important if conclusions about the potential binding sites of $S A \alpha 2,3$ binding viruses, such as influenza or human parainfluenza are to be made.
\end{abstract}




\section{Background}

There are two main epithelial cell types in the bronchus ciliated cells and goblet cells that secrete mucus. Within the submucosa there are also submucous glands present. The goblet cells have a glycoprotein which is acidic due to the presence of sialic sulphate groups and this secretion may vary with various diseases [1]. SA 2 2,6Gal has been reported to be present on the apical surface of ciliated cells but there have been conflicting reports about SA 2,3Gal expression on cell types. In cultured epithelial cells SA 2 ,6Gal appears to be present on non-ciliated (goblet) cells while $\mathrm{SA} \alpha 2,3 \mathrm{Gal}$ is present on ciliated cells [2]. On the contrary, others have reported that $S A \alpha 2,3 \mathrm{Gal}$ expression is found in goblet cells [3]. In addition, the patterns of glycosylation and the expression profile of SA 2,6 on cell surfaces may change during the course of developmental differentiation and following oncogenesis. For instance, SA $2,6 \mathrm{Gal}$ binding is weak during the glandular stage of lung development but increases as the lung matures [4]. Furthermore, if cells are exposed to inflammation and tumour necrosis factor there may be qualitative changes in glycosylation and the glycosyltransferases that lead to sialylation [5]. Recent publications, however, have indicated that both SA $\alpha 2,3 \mathrm{Gal}$ and SA $\alpha 2,6 \mathrm{Gal}$ may be present in the respiratory tract but with different distributions [6,7]. The presence or absence of these SA is important as human influenza A strains have been reported previously to preferentially attach to cells with SA $\alpha 2,6 \mathrm{Gal}$ linkages and avian strains preferentially bind SA 2 2,3Gal [8].

The affinity of the attachment of the HA to cell surface receptors is believed to be an important determinant in tissue tropism of the virus and constitutes part of the species barrier that keeps avian influenza viruses from readily infecting humans. Pigs contain a respiratory epithelium that has been reported to contain both "avian-virus" binding SA $2,3 \mathrm{Gal}$ and "human-virus" binding SA 2,6Gal linkages supposedly explaining why they can be infected with both human and avian influenza viruses [9]. Therefore pigs have been regarded as a hypothetical "mixing" vessel where re-assortment of avian and human viruses can take place, potentially leading to the emergence of pandemic influenza [9]. Given the presumed importance of the affinity of the influenza virus for its receptor, the distribution of SA $2,6 \mathrm{Gal}$ and SA $\alpha 2,3 \mathrm{Gal}$ expression in the human respiratory tract is critically important for the understanding of influenza pathogenesis.

Some of the studies on the types of SA expressed on cell surfaces have been done on sialic acids extracted from cell membrane homogenates. However, for the understanding of influenza pathogenesis and pandemic emergence, it is important to have methods that can define the profiles of SA $\alpha 2,6 \mathrm{Gal}$ and SA $\alpha 2,3 \mathrm{Gal}$ in histological tissue sec- tions in situ. The SA $\alpha 2,6 \mathrm{Gal}$ and SA $\alpha 2,3 \mathrm{Gal}$ expression on histological specimens in situ can be done using fluoresceinated lectins and by histochemistry. Since the early 1990's many histology laboratories have used unmasking or retrieval techniques to enhance immunohistochemical detection of antigens.

In this study we address three issues. The first was to investigate if antigen unmasking or retrieval would affect lectin-ligand expression in histological tissues. The second issue was to compare the findings obtained by using lectin fluorescence with cytochemistry for lectin-ligand analysis in the same tissue samples. Finally, we wanted to use optimized methods to re-evaluate the distribution of the SA $\alpha 2,6 \mathrm{Gal}$ and SA $\alpha 2,3 \mathrm{Gal}$ in human respiratory tissues and then correlate this with the reported presence or absence of influenza infection in different parts of the respiratory tract.

\section{Methods}

Biopsy samples were collected from the archived files of the Histopathology Department of Queen Mary Hospital, Pok Fu Lam, Hong Kong. Five surgically removed lungs from children with congenital cystic adenomatoid malformation (CCAM), seven non-neoplastic bronchial biopsies from patients investigated for possible malignancy, eight normal nasopharyngeal biopsies from patients with suspected nasopharyngeal carcinoma and eight lung biopsy samples from 20-40 week abortuses were also used. Seven biopsy tissues of CCAM, representing paediatric lung tissues of ages 1 month to 7 years were also retrieved from the files of the Department of Histopathology, Adelaide Women's and Children's Hospital, Adelaide, South Australia. All tissues had been fixed in 10\% neutral buffered formalin, processed into paraffin and stored at room temperature. Tissue blocks from the lungs of five patients who had died of acute bacterial pneumonia were used as a non-influenza comparison. Intestinal tissue from four ducks kindly provided by the Agriculture, Fisheries and Conservation Department, Government of HKSAR, were used as a positive control for SA 2 2,3Gal binding. The research was approved by the Ethics Committee of the University of Hong Kong/Hospital Authority Western Cluster.

We used lectin histochemistry and fluorescence which is the standard method of detection of the SA linkages [10]. Lectin analysis was performed using the fluorescein labelled lectins Sambucus nigra agglutinin (SNA) which primarily detects 6-linked sialic acids and Maackia amurensis agglutinin (MAA) which primarily identifies 3linked sialic acids. Both fluorescein isothiocyanatelabelled (FITC) and tetramethyl rhodamine isothiocyanate-labelled (TRITC) were used as fluorochromes and purchased from EY Laboratories (San Mateo, California). 
Additional FITC conjugated MAA1 was purchased from Vector Laboratories (Burlingame, CA). When peroxidase or biotin conjugation was used the conjugates were purchased from EY Laboratories (SNA and MAA) and Vector (MAA1 and MAA2). Digoxigenin labelled SNA and MAA was purchased from Roche as part of the Dig-Glycan Detection Kit.

For the initial trial of optimization for oligosaccharide ligand retrieval methods one lung block from a case of CCAM was used. The tissues were sectioned at $5 \mu \mathrm{m}$ and deparaffinized. Control sections had no retrieval. Two different methods were used for retrieval: microwave and enzyme digestion. For microwaving, an Energy Beam Sciences microwave processor was used together with 2 types of buffer. 0.1 M EDTA and $10 \mathrm{mM}$ citrate buffer was used and the sections were microwaved for 10,15, 20 and 25 minutes at $95^{\circ} \mathrm{C}$. Two enzyme digestion methods were used: trypsin and pronase, and for both these methods sections were incubated for 15 mins at $37^{\circ} \mathrm{C}$.

Single fluorescent studies were performed as follows. The sections were microwaved in $95^{\circ} \mathrm{C}$ citrate buffer $\mathrm{pH} 6.0$ for 15 minutes, washed with $0.05 \mathrm{M}$ Tris Buffer Saline (TBS) pH 7.6 and then incubated with either 1/100 FITC conjugated SNA (EY Laboratories), or 1/100 FITC conjugated MAA (EY Laboratories) for 1 hour at room temperature in the dark. Double fluorescent studies were performed according to the method of Mason et al. [11]. Briefly, the sections were microwaved in $95^{\circ} \mathrm{C}$ citrate buffer pH 6.0 for 15 minutes, washed with $0.05 \mathrm{M}$ Tris Buffer Saline (TBS) pH 7.6 and then incubated with 1/100 TRITC conjugated SNA (EY Laboratories) and 1/100 FITC conjugated MAA(EY Laboratories) for 1 hour at room temperature in the dark. The sections were washed with TBS 3 times for 5 minutes each and the nuclei stained with $5 \mu \mathrm{g} / \mathrm{ml}$ DAPI for 4 minutes followed by three washes with TBS of 5 minutes each and mounting with DAKO fluorescent mount (Dako, Glostrup, Denmark). Fluorescent examination was with a Nikon Eclipse microscope with SPOT Pursuit Camera (Sterling Heights, MI) and ImagePro Plus software (MediaCybernetics, MD) was used.

Lectin horseradish peroxidase (HRP) detection: the sections were microwaved in $10 \mathrm{mM}$ citrate buffer $\mathrm{pH} 6.0$ for $15 \mathrm{~min}$, blocked with $3 \% \mathrm{H}_{2} \mathrm{O}_{2}$ in TBS for $12 \mathrm{~min}$ and after washing with TBS 3 times, 5 minutes each were then incubated with 1/50 HRP conjugated SNA (EY Laboratories) and 1/50 HRP conjugated MAA (EY Laboratories) at room temperature for 1 hour respectively. After 3 further washes in TBS the sections were developed with AEC substrate kit (Vector Laboratories) at RT for 30 minutes followed by counterstaining with Mayer's haematoxylin and mounting with DAKO aqueous mount (Dako, Glostrup, Denmark).
Lectin biotin detection: The sections were microwaved in $10 \mathrm{mM}$ citrate buffer $\mathrm{pH} 6.0$ at $95^{\circ} \mathrm{C}$ for $15 \mathrm{~min}$ then blocked with $3 \% \mathrm{H}_{2} \mathrm{O}_{2}$ in TBS for $12 \mathrm{~min}$ and with avidin/ biotin blocking kit (Vector). They were then incubated with 1/100 biotinylated MAA1 (or 1/100 biotinylated MAA2) (Vector) for either 1 hour at RT or $4^{\circ} \mathrm{C}$ overnight, blocked with $1 \%$ bovine serum albumin for $10 \mathrm{mins}$ at $\mathrm{RT}$, and then incubated with strep-ABC complex (Dako Cytomation, K-0377) diluted 1/100 for 30 mins. at room temperature. Development was performed using the AEC substrate kit (Vector) at room temperature for 10 minutes. The nuclei were counterstained with Mayer's hematoxylin and then the sections were dried and mounted with DAKO aqueous mount (Dako Cytomation). Duck intestine sections were used as controls with and without pretreatment with SA $\alpha 2,3$ specific neuraminidase from Glyko to ensure that sialic acids were specifically targeted. Stain intensity was measured semi-quantitatively using duck MAA as a control. Similar stain intensity to the duck intestine was graded as strong $(++)$ and a weaker pattern as + .

To determine lectin binding profiles we used data from the Consortium for Functional Glycomics (CFG) web site [12] using glycan array data for MAA1(also known as MAL), SNA and a human H5N1 virus, (A/Vietnam/1203/ 04).

\section{Results}

\section{Microwave retrieval increased lectin binding}

In the absence of unmasking techniques and using the lectins SNA and MAA from EY Labs, there was minimal to weak (-/+) SNA binding and weak (+) MAA binding in the basal epithelium and epithelial cells of the bronchial mucosa of paediatric tissues. All forms of retrieval enhanced the lectin binding to the surface epithelial cells and mucus containing cells for both SNA and MAA (Figure 1) and this appeared to be most prominent in the submucous glands. Trypsin and pronase digestion also produced increased binding, but it also tended to result in more surface epithelial denudation of cells from the section. When microwave heating was used the increase in staining was maximal after 15 minutes. As citrate buffer heating appeared to just as beneficial as other forms of retrieval this was chosen as the preferred method of unmasking for future work. Tissue sections of duck intestine was used as a control and this showed MAA binding on the surface of the columnar cells in keeping with its SA 2 ,3Gal moiety.

\section{There is different binding of MAA isoforms to the upper and lower respiratory tract in adult and paediatric tissues} The 8 samples of fetal tissue at 20 weeks gestation showed strong binding $(++)$ of MAA to the bronchial epithelium and to the developing pneumocytes with absent SNA 

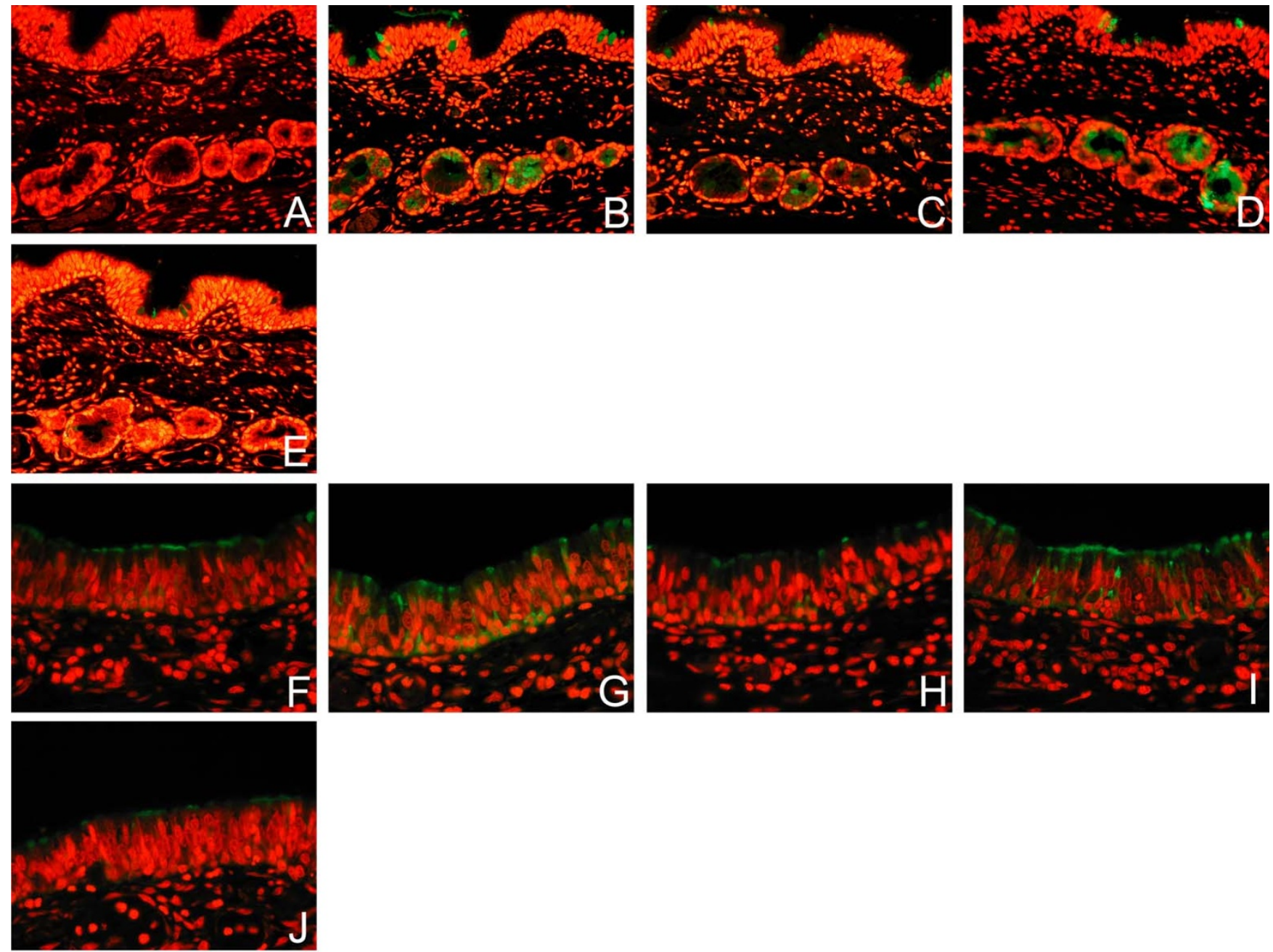

Figure I

Effect of different retrieval techniques on lectin binding. Single labelling of paediatric respiratory mucosa by FITC- conjugated Sambucus nigra agglutinin (SNA) (A-F) and Maackia amurensis agglutinin (MAA) (G-J) using different methods of retrieval. No antigen retrieval, $(A)$ and $(F)$, Citrate buffer $(B)$ and $(G)$, EDTA $(C)$ and $(H)$, Pronase $(D)$ and $(I)$, and Trypsin $(E)$ and $(J)$. Stain intensity was graded as strong $(++)$ and a weaker pattern as + . In the absence of unmasking techniques there was minimal to weak (-/+) SNA binding and weak (+) MAA binding in the basal epithelium and epithelial cells of the bronchial mucosa of paediatric tissues. All forms of retrieval enhanced the lectin staining of the surface epithelial cells and mucus containing cells for both SNA and MAA. Examination with dual FITC/Rhodamine filter. Magnification $\times 200$.

detected. With advancing development the SNA binding increased and there was a switch in the MAA1 and MAA2 binding between the alveoli and bronchi (Table 1). The paediatric tissues from children with CCAM showed strong $(++)$ binding in all cases of MAA to the bronchial epithelium but not to the alveoli. SNA was also strongly $(++)$ bound to the bronchial epithelium but there was weaker (+) binding to the alveoli. Because we found greater binding of MAA in the respiratory tract than previously reported using the MAA from one supplier (EY Laboratories) we tested the MAA from another supplier (Vector) to verify the results. The MAA from EY labs has been identified as a combination of 2 isoforms of MAA -
MAA1 and 2 which though both identifying SAa2,3Gal have different recognition patterns for the inner fragments. While MAA2 is specific towards SA $\alpha 2,3 \mathrm{Gal} \beta 1$, 3GalNAc and has been used to detect the "traditional" avian influenza receptor, MAA1 is more specific towards SA $\alpha 2,3 \mathrm{Gal} \beta 1,4 \mathrm{GlcNAc}[13]$. When an analysis of sequential sections from the upper and lower respiratory tract was performed a number of consistent findings were observed. Firstly, SNA binding was more widespread in the upper than the lower respiratory tract and this was more pronounced in the adult tissues (Fig 2A,D, G,J,M). It was also present in the mucus secreting cells as well as the ciliated cells with a strong intensity $(++)$. In the adult lung 
Table I: Summary of lectin binding intensities in fetal, paediatric and adult respiratory tissues using Sambucus nigra agglutinin (SNA) and Maackia amurensis $I$ and 2 (MAAI and MAA2).

\begin{tabular}{|c|c|c|c|c|c|c|c|c|c|c|c|c|}
\hline & \multicolumn{2}{|c|}{ Fetus $(n=8)$} & \multicolumn{3}{|c|}{ Paediatric lung $(n=12)$} & \multicolumn{2}{|c|}{ Adult NP $(n=8)$} & \multicolumn{2}{|c|}{$\begin{array}{l}\text { Adult bronchus } \\
\quad(\mathrm{n}=7)\end{array}$} & \multicolumn{3}{|c|}{ Adult lung $(n=5)$} \\
\hline & Alv & Bronch & Alv & Bronch & Gland & Epith & Gland & Epith & Gland & Macro & Pneu & Bronch \\
\hline SNA & $-\rightarrow+$ & $-\rightarrow+$ & + & $+/-$ & + & ++ & ++ & ++ & ++ & + & + & + \\
\hline MAAI & $++\rightarrow-$ & $-\rightarrow+$ & - & ++ & + & ++ & ++ & ++ & ++ & $+/++$ & - & ++ \\
\hline MAA2 & $-\rightarrow+$ & $+\rightarrow-$ & ++ & - & $+/-$ & - & - & - & - & - & ++ & - \\
\hline
\end{tabular}

Alv $=$ alveolus, Bronch $=$ bronchial epithelium, Epith $=$ epithelium, Macro $=$ macrophage and Pneu $=$ pneumocyte. The arrows in fetal tissue indicate the changes in expression from 20 weeks gestation to term.

the pneumocytes showed only weak binding $(+)$ of SNA (Fig $2 \mathrm{G}$ ) but there appeared to be stronger binding $(++)$ to the epithelium of the lungs of children (Fig $2 \mathrm{M}$ ). Secondly, MAA1 was widely bound throughout the all the respiratory tract of children and adults and did not vary with age after delivery (Fig 2B,E,H,K,N). It bound to ciliated cells as well as the mucus secreting cells. In the paediatric bronchus it also bound to the basal cells (Fig $2 \mathrm{~K}$ ). Thirdly, MAA 2 binding appeared to be limited only to pneumocytes $(+-++)$ and did not bind to cells in the nasopharynx or the bronchial epithelium (Fig 2F,O).

\section{Within the adult bronchus there was heterogeneous distribution of lectin binding}

When dual labelling was performed on the bronchial tissues of adults and of children using SNA and MAA, we identified a heterogeneous distribution of SNA and MAA binding in the epithelium with no clear distinction between ciliated and non-ciliated cells resulting in a mixed and occasionally dual expression of both SA 2,3Gal as well as $S A \alpha 2,6 \mathrm{Gal}$ in the ciliated cells, goblet cells and basal cells (Fig 3A). We also found a similar staining pattern when non-fluorochromes were used (Fig 3B-E).

\section{The MAA from different suppliers has different binding patterns}

Because we found that there was a different binding pattern in the upper and lower respiratory tract for MAA1 and MAA2 using these lectins from Vector labs we then investigated whether the MAA from 2 other suppliers - EY Laboratories and Roche would identify one or both of the MAA isoforms. We therefore used serial sections from adult lung tissue and stained them using the Vector MAA1 and MAA2, digoxigenin labelled MAA (Roche) and biotinylated MAA from EY Laboratories. While EY Laboratories state that their MAA is a combination of 2 isoforms, this information is not evident from Roche. As expected using MAA1 (Fig 4A,E) the alveolar macrophages (orange arrows) and bronchial cells (blue arrow) bound MAA but pneumocytes (green arrow) showed no binding. Conversely MAA2 (Fig 4B,F) bound to pneumocytes (green arrows). The digoxigenin labelled MAA from Roche (Fig $4 \mathrm{C}, \mathrm{G})$ mainly bound to macrophages (orange arrows) and bronchial cells (blue arrow) but there was minimal binding to pneumocytes (green arrows) indicating that this MAA is mainly identifying MAA1 rather than MAA2. The MAA from EY Laboratories (Fig 4D,H) showed a weak $(+)$ binding to the pneumocytes (green arrows) as well as strong binding $(++)$ to bronchial epithelial cells and macrophages but it should be noted that this binding to pneumocytes appeared weaker than MAA2.

Analysis of the glycan array binding data of MAA1(also known as MAL), SNA and H5N1 submitted to the CFG showed that MAL did detect SA $2,3 \mathrm{Gal} \beta 1,4 \mathrm{GlcNac}$ (glycan numbers 235-238) with detection of the sulphated forms (216 and 227) [see Additional file 1]. There was absent binding to SA $2,3 \mathrm{Gal} \beta 1,3 \mathrm{GalNAc}$ (number 202). If a reading of 2000-4000 is taken as a weak binding then MAL also had weak binding to $S A \alpha 2,6 \mathrm{Gal} \beta 1,4 \mathrm{Glc}$ (number 249) but not when the Glc was replaced by GlcNAc (numbers 245,246). As expected, the H5N1 (Viet04) virus bound strongly to SA $\alpha 2,3 \mathrm{Gal}$ termini and many of the strong virus- binding oligosaccharides were identified by MAA1 (e.g. numbers 235-237). While MAA2 or (MAH) has not been analysed in glycan arrays, it preferentially detects the O-linked SA $\alpha 2,3 \mathrm{Gal} \beta 1,3$ [SA 2,6$] \mathrm{Gal}-$ NAc [13]. This glycan (221) shows strong binding for H5N1 but minimal binding for MAA1 [see Additional file 1]. MAA1 does show high affinity to non-sialic acid residues especially those with a sulphate moiety (e.g. glycan numbers 28-38) which has led to it not being recommended for use for identifying the SA $\alpha 2,3$ terminated oligosaccharides and indeed we found that using neuraminidase treatment of sections that MAA1 and 2 binding could be abolished in duck intestine tissues but trials in human tissues did show residual MAA1 binding after treatment (data not shown).

\section{Discussion}

Cells of the respiratory tract express a number of glycan containing conjugates on the cell surface, many of which terminate with $N$-acetylneuraminic (sialic, SA) acids - a 


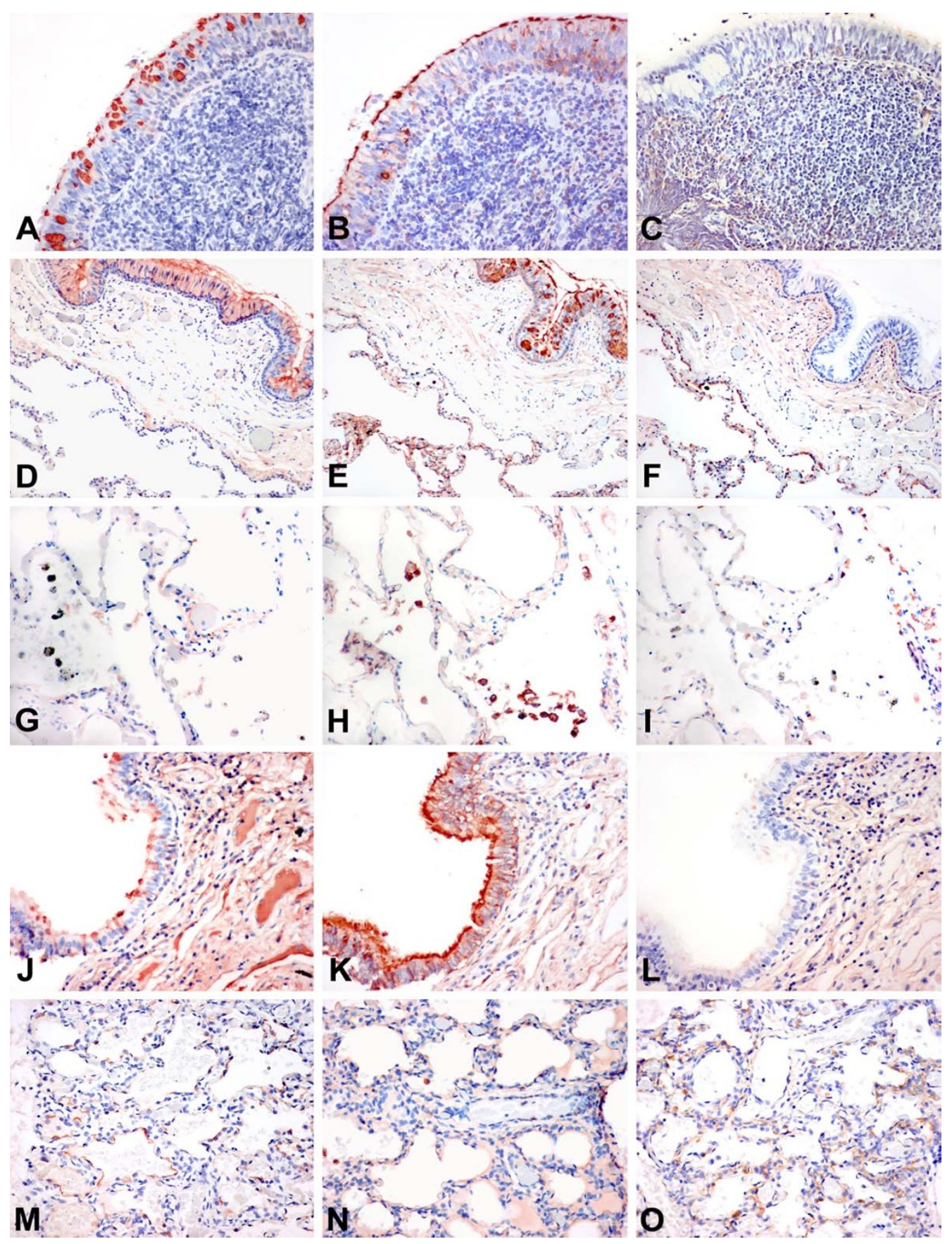

\section{Figure 2}

Lectin binding to upper and lower respiratory tract. Tissue distribution of Sambucus nigra agglutinin (SNA) for SA $\alpha 2,6$, Maackia amurensis agglutinin I (MAAI), and Maackia amurensis agglutinin 2 (MAA2) for SA 2 2,3 binding in the adult and paediatric respiratory tract. Serial sections of nasopharynx (A-C), adult bronchus (D-F), adult lung (G-I), paediatric bronchus (J-L), and paediatric lung (M-O) are shown and stained with SNA (A,D,G,J,M), MAAI (B,E,H,K,N) and MAA2 (C,F,I,L,O). The adult nasopharynx shows SNA and MAAI binding in the epithelium but no MAA2 binding. A similar pattern is also present in the adult bronchus and in addition the pneumocytes show MAAI and MAA2 binding (E,F). Alveolar macrophages (G-I) demonstrate minimal SNA and no MAA2 binding but are positive for MAAI. The paediatric bronchus shows a greater binding of the epithelium with MAAI $(K)$ than the adult $(E)$. The pneumocytes $(M)$ also show more SNA binding than the adult (G). Staining using HRP conjugated SNA and biotin conjugated MAAI and MAA2. (A-F) and (J-L) at $200 \times$ magnification and (G-I) and (M-O) at $400 \times$ magnification. 

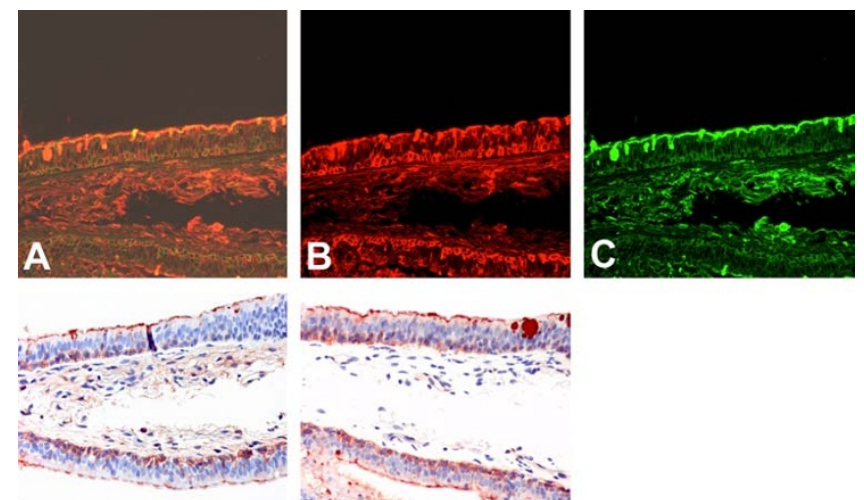

\section{D}

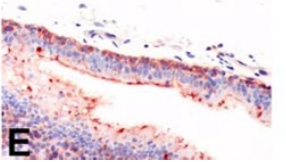

Figure 3

Lectin binding to bronchial epithelium using different detection methods. Serial sections for comparison of detection methods for lectin binding of Sambucus nigra agglutinin (SNA) to SA 2,6 and Maackia amurensis (MAA) agglutinin for binding to $S A \alpha 2,3$ in adult bronchial epithelium. Double fluoresecence for FITC labelled SNA and TRITC labelled MAA shows a heterogeneous pattern $(A)$, with more binding to the basal epithelium with MAA (B) than SNA (C). HRP labelling of MAA (D) and SNA (E) shows a similar pattern of binding to the fluorescent labelled lectin. $200 \times$ magnification.

series of 9-carbon sugars. Influenza virus infection of humans involves binding of the virus haemagglutinin (HA) to these sialyoligosaccharides on the surface of cells of the respiratory tract. In addition, the virus neuraminidase (NA) cleaves the sialic acid on the host cell and is important in releasing newly formed virus from the cell after virus replication is completed so these virions can spread out in search of other cells to infect. Since respiratory mucus is also rich in SA, this provides a potential barrier to the spread of newly formed virions. By cleaving these SA, the influenza virus NA facilitates the virus spread through this mucus layer [14]. Thus the balance between the affinity of binding between the virus and the cell receptor and the virus-releasing activity of the NA is critical to virus replication in a host species.

The crystal structures of HA shows that the terminal sialic acids bind in a groove at the top of the HA molecule [15]. Previous investigations had indicated that avian viruses would preferentially bind $S A \alpha 2,3 \mathrm{Gal}$ and human viruses $\mathrm{SA} \alpha 2,6 \mathrm{Gal}$ [8]. The avian and human $\mathrm{H} 5 \mathrm{~N} 1$ viruses causing the "bird flu" outbreak in Hong Kong in 1997 had

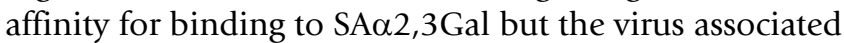
with human disease in Hong Kong in 2003 had affinity to bind to both avian-like SA 2,3Gal and human-like SA 2,6Gal [16]. H5N1 disease in humans has been reported to be different from conventional human influenza viruses (H3N2 or H1N1) in that the lower (rather than upper) respiratory tract is the major target for virus replication [7].

Using retrieval methods and selection of lectin conjugate we have demonstrated that the lectin binding to the SA $\alpha 2,3 \mathrm{Gal}$ receptor for avian influenza viruses is more

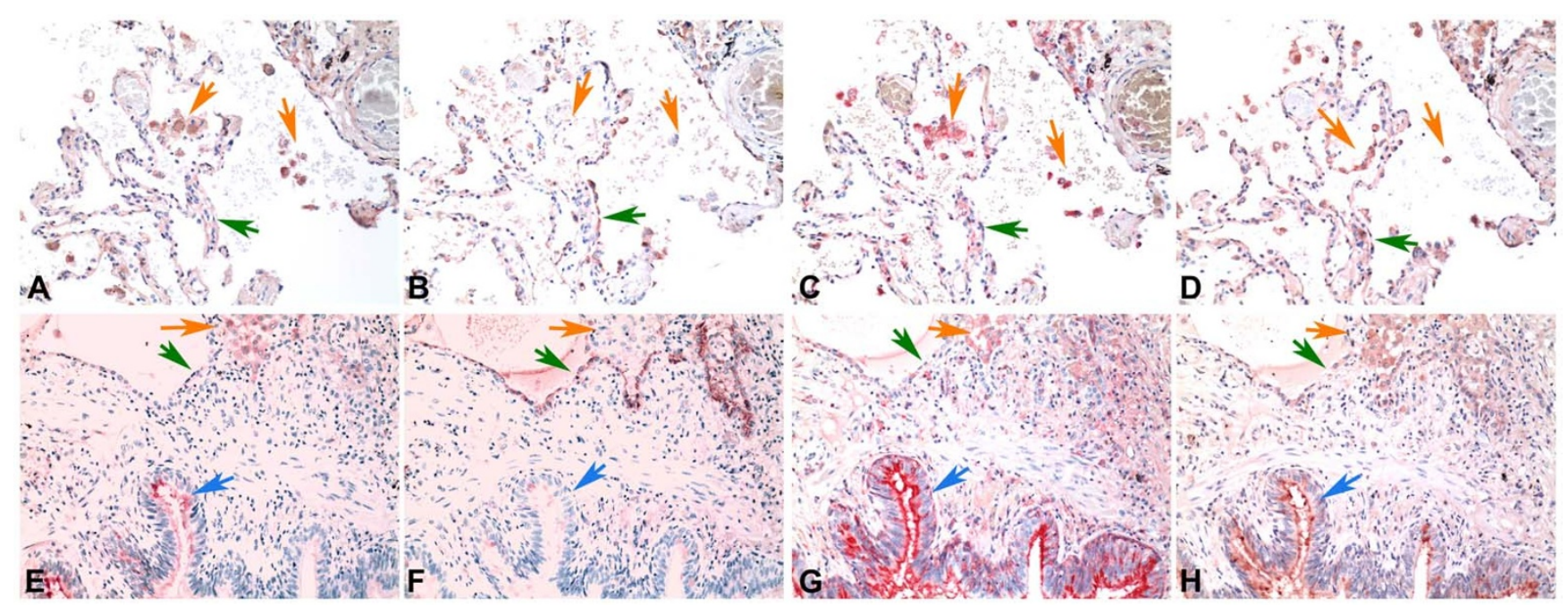

\section{Figure 4}

Comparison of MAA binding using MAA from different suppliers. Serial sections of adult lung tissue for comparison of lectin binding of Maackia amurensis (MAA) for SA 2 2,3Gal. Biotin conjugated MAAI (also known as MAL) from Vector Laboratories $(A)$ and $(E)$, Biotin conjugated MAA2 (also known as MAH) from Vector Laboratories $(B)$ and $(F)$, Digoxigenin conjugated MAA from Roche $(C)$ and $(G)$ and HRP conjugated MAA from EY Laboratories $(D)$ and $(H)$. Orange arrows indicate alveolar macrophages, Green arrows indicate alveolar pneumocytes and blue arrows indicate bronchiolar epithelium. Haematoxylin counterstain $200 \times$ magnification. 
widely expressed in the respiratory tract of humans than previously documented [7]. In particular, we demonstrated that unlike the previous reports that indicated certain type of cells had only one lectin binding profile, SA 2,6 and SA 2 2,3Gal was found in ciliated epithelium, goblet cells and submucous glands in the bronchus as well as pneumocytes of the alveoli, and SA 2,3 was also present in the metaplastic epithelium. In keeping with an earlier report [4], we found that neonatal pneumocytes expressed mainly SA $\alpha 2,3 \mathrm{Gal}$ and the neonatal bronchus was also primarily $S A \alpha 2,3 \mathrm{Gal}$ expressing. The respiratory tract of young children showed mainly SA $2,3 \mathrm{Gal}$ with a lower level of $S A \alpha 2,6 \mathrm{Gal}$ expression than adult tissues. This may, in part explain why children appear to be more susceptible to avian influenza $\mathrm{H} 5 \mathrm{~N} 1$ in the recent outbreaks in East Asia.

The difference between our studies and previous ones on sialic acid expression in respiratory epithelial cells can be partially explained by the methods used for lectin analysis as well as the type of lectin conjugate used. Antigen retrieval or unmasking did not become an established procedure in many laboratories until the mid 1990's and the earlier publications used paraffin embedded tissues without retrieval $[3,8]$. Later studies on pigs, primates and ducks also did not use retrieval procedures $[9,17]$. While the precise mechanism of retrieval still remains not precisely defined the general consensus is that heating or the use of enzymes serves to unmask antigenic sites that have become cross-linked through formalin fixation [18]. The unmasking of epitopes appears to extend to carbohydrate moieties as well as proteins. The increased detection of SA $2,6 \mathrm{Gal}$ through unmasking has also been recently shown in the liver [19].

Two isoforms of MAA have been recognized for many years and their binding profiles have been characterized. Since MAA1 bound to non-SA 2,3 glycans it has not been used as extensively by some researchers as MAA2. For instance, Shinya et al have recently demonstrated SA 2,3 Gal expression only in the lung but not the bronchus or upper respiratory tract [7]. Since they only used MAA2 lectin binding (Y. Kawaoka, personal communication) our MAA2 results are in accord with theirs. But our finding of MAA1 binding in the upper and lower respiratory tract does have implications for the possible distribution of receptors for avian influenza viruses including the currently circulating $\mathrm{H} 5 \mathrm{~N} 1$ viruses. The crucial question therefore is whether for the identification of susceptible binding sites for avian influenza viruses which are known to bind SA 2,3Gal, should researchers should just use MAA2 or should they also use MAA1? While both of these isoforms do identify the $S A \alpha 2,3 \mathrm{Gal}$ ending they differ in their affinity for the inner fragments of the glycans, and these inner fragments are known to affect different virus binding [20]. To answer this we used glycan array data performed by the CFG for a known H5N1 avian influenza virus (Viet04) and also the affinity data for MAA1 (also known as MAL) [12]. As expected Viet 04 does have strong affinity for SA $2,3 \mathrm{Gal}$ terminated glycans and that MAL does identify SA $\alpha 2,3 \mathrm{Gal}$ glycans but it also identifies nonSA 2,3 moieties and there is an overlap in the glycans that Viet 04 and MAA1 have high affinity to [see Additional file 1]. Thus, there are H5N1 binding SA 2,3 terminated oligosaccharides that are detected with MAA1 (e.g. glycans 235-237), but these potential binding sites may not be detected if only MAA2 is used. We therefore believe that MAA1 should be used in conjunction with MAA2 in determining tissue distribution but do acknowledge that as it does detect non-SA terminated residues ancillary methods such as neuraminidase treatment of sections may be needed. Furthermore our findings that the MAA supplied by Roche and EY Laboratories primarily identifies MAA1 also indicates that these lectins should be used in conjunction with specific lectins from Vector labs to avoid misinterpretation of potential binding sites for $\mathrm{SA} \alpha 2,3$ binding viruses. It is of note that our findings are applicable not only to influenza viruses but also to some parainfluenza

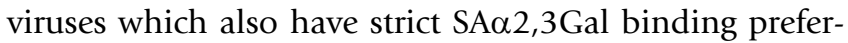
ence.

Because of this varied distribution of MAA1 and MAA2 throughout the respiratory tract, we hypothesised that this may shed new light on the distribution and binding of H5N1 viruses to the upper and lower respiratory tract. Using this information we therefore used ex-vivo cultures of the upper and lower respiratory tract and infected them with different H5N1, H1N1 and H3N2 viruses and found that contrary to previous suppositions, H5N1 viruses were able to replicate in the upper respiratory tract - a region which lacked MAA2 binding but had abundant MAA1 binding, thus indicating that the virus is perhaps binding to SA $2,3 \mathrm{Gal} \beta 1,3 / 4 \mathrm{GlcNac}$ motifs or even to non-sialyated receptors [21].

Our results also indicated that the recent findings of Matrosovich and colleagues who found SA $2,3 \mathrm{Gal}$ in ciliated cells and $S A \alpha 2,6 \mathrm{Gal}$ in the goblet cells of tracheobronchial cells cultured in vitro are only a partial representation of the true nature of sialic acid expression in the adult respiratory tract [2]. Their findings in the in vitro model with cultured tracheobronchial epithelium showed more similarity to the receptor profile seen in the respiratory tract of children. Therefore it is possible that the human tracheobronchial epithelial culture model is more representative of the respiratory tract of children rather than that of adults, and represents a developmentally earlier model of the human respiratory tract. 


\section{Conclusion}

In summary, we found that there was an overall increased detection of $\mathrm{SA} \alpha 2,3 \mathrm{Gal}$ in the respiratory tract when microwave unmasking is used and when a combination of different isoforms of MAA was used. We therefore advocate the routine use of this method for future investigations on the distribution of receptors for influenza viruses in the respiratory tract. We also urge attention to the exact isoform of MAA present in lectins supplied by different manufacturers [22]. These results imply a need for a reevaluation of the findings reported in previous studies on the tissue distribution of SA receptor types. Furthermore, it appears that tissues of children have a greater expression of SA $2,3 \mathrm{Gal}$ than previously described, potentially conferring a greater susceptibility avian influenza viruses. Finally we were able to use the lectin histochemical findings to re-evaluate the tissue tropism of $\mathrm{H} 5 \mathrm{~N} 1$ infection of the respiratory tract and shed new light on the cells infected by this emerging virus by judicious used of both isoforms of MAA.

\section{Competing interests}

The author(s) declare that they have no competing interests.

\section{Authors' contributions}

Dr J Nicholls and Professor J S M Peiris designed the experiments and were responsible for primary analysis of tissues. Dr A J Bourne provided input to the CCAM cases from Australia. Dr H Chen and Y Guan assisted in the interpretation of results. All authors have read and approved the final manuscript.

\section{Additional material}

\section{Additional file 1}

Maackia amurensis 1 (MAA1), Sambucus nigra agglutinin (SNA) and H5N1 binding affinity in glycan array Description: Summary of glycan binding profiles of Maackia amurensis 1 (MAA1), Sambucus nigra agglutinin (SNA) and H5N1 influenza (A/Vietnam/1203/04) (Viet04) in the glycan array. Significant binding sugars are shown in column 2 with their glycan numbers according to Printed Array 2.1 shown in column 1.

Strong affinity is **, weak affinity is * and no significant affinity is blank. Many SA 22,3Gal oligosaccharides (underlined) bind to both MAA1 as well as H5N1 (A/Vietnam/1203/4).

Click here for file

[http://www.biomedcentral.com/content/supplementary/14659921-8-73-S1.pdf]

\section{Acknowledgements}

Kevin Fung, Chan Yuk Sing and Joanne Lo for technical assistance. Funding provided by RFCID from the Government of HK SAR (03040872) and Wellcome Trust, UK (08/ I84/Z/06/Z).

\section{References}

I. Lamblin G, Lhermitte M, Klein A, Roussel P, Van Halbeek H, Vliegenthart JF: Carbohydrate chains from human bronchial mucus glycoproteins: a wide spectrum of oligosaccharide structures. Biochem Soc Trans 1984/08/0I edition. 1984, I 2(4):599-600.

2. Matrosovich MN, Matrosovich TY, Gray T, Roberts NA, Klenk HD: Human and avian influenza viruses target different cell types in cultures of human airway epithelium. Proc Natl Acad Sci U S A 2004/04/09 edition. 2004, I 0 I (13):4620-4624.

3. Baum LG, Paulson JC: Sialyloligosaccharides of the respiratory epithelium in the selection of human influenza virus receptor specificity. Acta Histochem Suppl 1990/0I/0I edition. 1990, 40:35-38.

4. Cerna A, Janega P, Martanovic P, Lisy M, Babal P: Changes in sialic acid expression in the lung during intrauterine development of the human fetus. Acta Histochem 2003/0I/30 edition. 2002, I 04(4):339-342.

5. Delmotte P, Degroote S, Merten MD, Van Seuningen I, Bernigaud A, Figarella C, Roussel P, Perini JM: Influence of TNFalpha on the sialylation of mucins produced by a transformed cell line MM-39 derived from human tracheal gland cells. Glycoconj J 2002/06/27 edition. 200I, I 8(6):487-497.

6. Barkhordari A, Stoddart RW, McClure SF, McClure J: Lectin histochemistry of normal human lung. J Mol Histol 2004/08/27 edition. 2004, 35(2): I47-I56.

7. Shinya K, Ebina M, Yamada S, Ono M, Kasai N, Kawaoka Y: Avian flu: influenza virus receptors in the human airway. Nature 2006/ 03/24 edition. 2006, 440(7083):435-436.

8. Rogers GN, Paulson JC: Receptor determinants of human and animal influenza virus isolates: differences in receptor specificity of the $\mathbf{H} 3$ hemagglutinin based on species of origin. Virology 1983/06/0 I edition. 1983, I27(2):36I-373.

9. Ito T, Couceiro JN, Kelm S, Baum LG, Krauss S, Castrucci MR, Donatelli I, Kida H, Paulson JC, Webster RG, Kawaoka Y: Molecular basis for the generation in pigs of influenza $A$ viruses with pandemic potential. J Virol 1998/08/08 edition. 1998, 72(9):7367-7373.

10. Suzuki Y: Sialobiology of influenza: molecular mechanism of host range variation of influenza viruses. Biol Pharm Bull 2005/ 03/04 edition. 2005, 28(3):399-408.

II. Mason DY, Micklem K, Jones M: Double immunofluorescence labelling of routinely processed paraffin sections. J Pathol 2000/08/05 edition. 2000, I 9 I(4):452-46I.

12. Glycomics TCF: - [http://www.functionalglycomics.org/glycomics/ publicdata/primaryscreen.jsp].

13. Konami Y, Yamamoto K, Osawa T, Irimura T: Strong affinity of Maackia amurensis hemagglutinin (MAH) for sialic acid-containing Ser/Thr-linked carbohydrate chains of $\mathbf{N}$-terminal octapeptides from human glycophorin A. FEBS Lett I994/04/I I edition. 1994, 342(3):334-338.

14. Wagner R, Matrosovich M, Klenk HD: Functional balance between haemagglutinin and neuraminidase in influenza virus infections. Rev Med Virol 2002/05/03 edition. 2002, I2(3): | 59-166.

15. Skehel J], Wiley DC: Receptor binding and membrane fusion in virus entry: the influenza hemagglutinin. Annu Rev Biochem 2000/08/3I edition. 2000, 69:53I-569.

16. Shinya K, Hatta M, Yamada S, Takada A, Watanabe S, Halfmann P, Horimoto T, Neumann G, Kim JH, Lim W, Guan Y, Peiris M, Kiso M, Suzuki T, Suzuki Y, Kawaoka Y: Characterization of a human H5N I influenza A virus isolated in 2003. J Virol 2005/07/I5 edition. 2005, 79(I 5):9926-9932.

17. Gagneux P, Cheriyan M, Hurtado-Ziola N, van der Linden EC, Anderson D, McClure H, Varki A, Varki NM: Human-specific regulation of alpha 2-6-linked sialic acids. J Biol Chem 2003/09/23 edition. 2003, 278(48):48245-48250.

18. Shi SR, Cote RJ, Taylor CR: Antigen retrieval techniques: current perspectives. J Histochem Cytochem 200 I/07/I8 edition. 200 I, 49(8):931-937.

19. Dall'Olio F, Chiricolo M, D'Errico A, Gruppioni E, Altimari A, Fiorentino $M$, Grigioni WF: Expression of beta-galactoside alpha2,6 sialyltransferase and of alpha2,6-sialylated glycoconjugates in normal human liver, hepatocarcinoma, and cirrhosis. Glycobiology 2003/09/30 edition. 2004, I4(I):39-49. 
20. Gambaryan A, Yamnikova S, Lvov D, Tuzikov A, Chinarev A, Pazynina G, Webster R, Matrosovich M, Bovin N: Receptor specificity of influenza viruses from birds and mammals: new data on involvement of the inner fragments of the carbohydrate chain. Virology 2005/03/23 edition. 2005, 334(2):276-283.

21. Nicholls JM, Chan MC, Chan WY, Wong HK, Cheung CY, Kwong DL, Wong MP, Chui WH, Poon LL, Tsao SW, Guan Y, Peiris JS: Tropism of avian influenza $A(H 5 N I)$ in the upper and lower respiratory tract. Nat Med 2007/0I/09 edition. 2007, I3(2): I47- I49.

22. Ibricevic A, Pekosz A, Walter MJ, Newby C, Battaile JT, Brown EG Holtzman MJ, Brody SL: Influenza virus receptor specificity and cell tropism in mouse and human airway epithelial cells. I Virol 2006/07/I5 edition. 2006, 80(15):7469-7480.

Publish with Bio Med Central and every scientist can read your work free of charge

"BioMed Central will be the most significant development for disseminating the results of biomedical research in our lifetime. "

Sir Paul Nurse, Cancer Research UK

Your research papers will be:

- available free of charge to the entire biomedical community

- peer reviewed and published immediately upon acceptance

- cited in PubMed and archived on PubMed Central

- yours - you keep the copyright

Submit your manuscript here:

http://www.biomedcentral.com/info/publishing_adv.asp
BiolMedcentral 